\title{
Decoupling and de Sitter vacua in approximate no-scale supergravities
}

\author{
M.C. David Marsh, ${ }^{a}$ Bert Vercnocke ${ }^{b}$ and Timm Wrase ${ }^{c}$ \\ ${ }^{a}$ Rudolf Peierls Centre for Theoretical Physics, University of Oxford, \\ 1 Keble Road, Oxford OX1 3NP, U.K. \\ ${ }^{b}$ Institute of Physics, University of Amsterdam, \\ Science Park, Postbus 94485, 1090 GL Amsterdam, The Netherlands \\ ${ }^{c}$ Stanford Institute for Theoretical Physics, Department of Physics, Stanford University, \\ 382 Via Pueblo Mall, Stanford, CA 94305-4060, U.S.A. \\ E-mail: david.marsh1@physics.ox.ac.uk, bert.vercnocke@uva.nl, \\ timm.wrase@stanford.edu
}

Abstract: We study $\mathcal{N}=1$ supergravity with $N>1$ chiral superfields in which one of the fields has a Kähler potential of exact no-scale type. Such systems admit de Sitter (dS) solutions in which supersymmetry is predominantly broken by the no-scale field, with only a small contribution to the breaking coming from the other fields. Metastable dS vacua of this type were recently shown to be achievable by the finetuning of an $N \times N$ sub-matrix of the Hessian matrix at the critical point. We show that perturbatively small deformations of the no-scale Minkowski vacuum into dS are only possible when the spectrum of the no-scale vacuum, besides the no-scale field, contain an additional massless mode. The no-scale structure allows for a decoupling of $N-2$ fields, and metastability can be achieved by the tuning of $\mathcal{O}(1)$ parameters. We illustrate this scenario in several examples, and derive a geometric condition for its realisation in type IIB string theory. Supergravities in which the complex structure moduli space is a symmetric space, such as the string theory inspired STU-models, are non-generic and realise a modified version of the scenario. For the STU-model with a single non-perturbative correction we present an explicit analytic family of dS solutions that includes examples with quantised fluxes satisfying the O3-plane tadpole condition.

KEYwords: Flux compactifications, dS vacua in string theory, Supergravity Models

ArXiv EPRINT: 1411.6625 


\section{Contents}

1 Introduction 1

2 Unbroken no-scale vacua 4

3 Approximate no-scale vacua $\quad 6$

$\begin{array}{lll}3.1 \text { Critical point equations } & 7\end{array}$

3.2 The perturbed mass-matrix $\quad 8$

3.2.1 Masses for the $X^{i}$ fields $\quad 8$

$\begin{array}{ll}\text { 3.2.2 Decoupling and stabilisation of the no-scale direction } & 10\end{array}$

4 Examples in supergravity and string theory $\quad 12$

4.1 Models with one non-perturbative effect: generalities 12

$\begin{array}{lll}4.2 & \text { Simple examples in supergravity } & 13\end{array}$

$\begin{array}{ll}4.3 & \text { Examples in type IIB string theory: generalities }\end{array}$

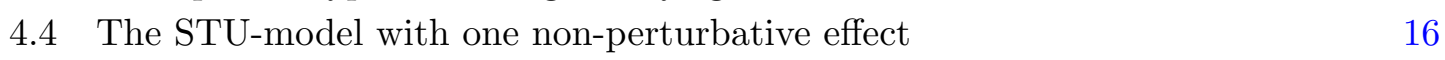

5 Conclusions 20

A Useful formulae $\quad 22$

A.1 STU-model 24

\section{Introduction}

The observation of an apparently accelerated expansion of the universe $[1,2]$ is perhaps the most striking discovery of modern cosmology. Recent, combined data from the Cosmic Microwave Background (CMB) and distant supernovae gives strong evidence for the energy density of the universe being dominated by a cosmological constant [3, 4], thus making the present universe well approximated by four dimensional de Sitter space.

String theory may accommodate a small positive cosmological constant through the numerous metastable solutions forming the 'landscape' of type IIB flux vacua, but determining the statistical properties of such vacua has proven challenging. While constructing fully generic flux vacua involving a large number of moduli is computationally prohibitive, much can be learned from subsets of solutions in which a substantial number of moduli can be made to decouple. In the KKLT scenario [5], complex structure moduli are supersymmetrically stabilised at a scale that is much larger than the scale of supersymmetry breaking, $m_{3 / 2}$, thus ensuring that these fields generically can be integrated out of the Wilsonian effective field theory and do not develop tachyonic directions upon supersymmetry breaking. In the Large Volume Scenario (LVS) [6, 7], complex structure moduli obtain masses of the order of $m_{3 / 2}$, but an underlying no-scale symmetry again leads to decoupling and 
a substantially more tractable low-energy dynamics, at least in the non-supersymmetric AdS minimum. ${ }^{1}$ In both KKLT and LVS, the final metastable de Sitter solution may be obtained by the inclusion of some additional, 'uplifting', source of supersymmetry breaking.

Recently, the construction of metastable de Sitter vacua through spontaneous supersymmetry breaking has received a lot of attention (see for example [9-25] and references therein). By constructing solutions that are consistently captured by spontaneously broken $\mathcal{N}=1$ supergravity, one can hope for improved computability and a more detailed picture of this part of the 'landscape'. Particularly interesting are those solutions which make use of an exact or approximate no-scale symmetry of the Kähler potential for some of the fields. No-scale Kähler potentials are commonly encountered in the dimensionally reduced, four dimensional effective theories arising from compactifications of string theory, and are in the simplest case of a single no-scale modulus $T$ and $N-1$ other moduli $X^{i}$ with $i=1, \ldots N-1$, given by,

$$
K=K_{\text {no-scale }}(T, \bar{T})+\tilde{K}\left(X^{i}, \bar{X}^{\bar{\imath}}\right)=-3 \ln (T+\bar{T})+\tilde{K}\left(X^{i}, \bar{X}^{\bar{\imath}}\right),
$$

while the superpotential is independent of $T$,

$$
W=W_{0}\left(X^{i}\right) .
$$

The perhaps most well-known example of such an effective theory is the dimensionally reduced type IIB compactification with a single Kähler modulus $T, N-2$ complex structure moduli, $U^{i}$, and the axio-dilaton $S$.

The F-term supergravity potential is remarkably simplified for no-scale models,

$$
V=e^{K}\left(F_{a} \bar{F}^{a}-3|W|^{2}\right)=e^{K} F_{i} \bar{F}^{i},
$$

where $a$ runs over all fields, $i$ runs over the $X^{i}$ fields, $F_{a}=D_{a} W=\left(\partial_{a}+K_{a}\right) W$ and we have used natural units to set $M_{\mathrm{Pl}}=2.4 \times 10^{18} \mathrm{GeV}$ to one. For $F_{i}=0$, as in the 'GKP' type IIB flux compactifications of [26], the $X^{i}$ fields are supersymmetrically stabilised and $T$ remains a flat direction of the Minkowski vacuum.

Recently, motivated by the numerical solutions of [20,21,27], reference [28] constructed a class of analytical de Sitter solutions in which supersymmetry is predominantly broken by the no-scale field $T$, with only a small amount of supersymmetry breaking in the perpendicular directions. For type IIB realisations of this mechanism, the smallness of the supersymmetry breaking in the directions perpendicular to $T$ can be achieved by tuning of fluxes, as discussed in [27]. A given critical point is a metastable minimum of the potential if all the eigenvalues of the $2 N \times 2 N$ hermitian Hessian matrix,

$$
\mathcal{H}=\left(\begin{array}{cc}
\partial_{a \bar{b}}^{2} V & \partial_{a b}^{2} V \\
\partial_{\bar{a} \bar{b}}^{2} V & \partial_{\bar{a} b}^{2} V
\end{array}\right),
$$

are positive. In [28], analytical conditions for the positivity of the eigenvalues of the diagonal $N \times N$ sub-matrix $\partial_{a \bar{b}}^{2} V$ were derived, and it was shown that upon tuning the

\footnotetext{
${ }^{1}$ For a recent discussion on the spectrum of the complex structure moduli in de Sitter space, see [8].
} 
$N \times N$ off-diagonal block $\partial_{a b}^{2} V$ to vanish or to be very small, the full Hessian could be made positive definite.

This type of solutions were shown to be realisable in string theory inspired 'STU' supergravity models with $N=3$, however, a general concern with this method is the significant fine-tuning needed to ensure metastability: for $N \gg 1$, the required $\mathcal{O}\left(N^{2}\right)$ fine-tuning of $\partial_{a b}^{2} V$ can be greatly limiting.

In this paper, we show that, given a small amount of no-scale breaking, there are approximate no-scale solutions that require only minimal, $\mathcal{O}\left(N^{0}\right)=\mathcal{O}(1)$, tuning to ensure the metastability of the Hessian matrix. To do this, we systematically expand the Hessian matrix, equation (1.4), in the small no-scale breaking parameter $\epsilon$. In particular we note that to zeroth order in no-scale breaking, $\partial_{a b}^{2} V$ is not small, yet has a structure that ensures that the mass matrix for all $X^{i}$ fields is semi-positive definite. This is a well-known property of flux compactifications of GKP type, that we here fully explore.

No-scale Minkowski vacua have at least two real massless fields corresponding to the flat directions along the complex field $T$. We find that, to zeroth order in $\epsilon$, the no-scale critical points that can be perturbed into de Sitter vacua in addition always have a third real massless mode in the spectrum of the fields $X^{i}$. This additional flat direction is generically lifted at linear order $\epsilon$, while the complex no-scale field $T$ is lifted at order $\epsilon^{2}$. The underlying no-scale symmetry ensures that the $2 N-3$ real fields that are lifted at order $\mathcal{O}\left(\epsilon^{0}\right)$ are metastable with positive definite masses. A remarkable decoupling of the heavy modes in the Hessian matrix at the approximately no-scale critical points then ensure that metastability can be achieved by the tuning of only two terms, independently of $N$.

As an illustration, we contrast these findings to those obtained in 'random supergravity' in which the superpotential and Kähler potential are taken to be random functions in the sense of [29], and in which only a fraction $P \lesssim \exp (-N)$ of the critical points are metastable minima. ${ }^{2}$ For the approximately no-scale critical points constructed in this paper, we show that the corresponding fraction is $1 / 2$, independently of $N$ and the value of the gravitino mass.

The general mechanism presented in this paper appears readily embeddable in flux compactifications of type IIB string theory, and we derive a sufficient condition on the complex structure field space geometry for the realisation of the mechanism with minimal $\mathcal{O}(1)$ fine-tuning. When the complex structure field space is a symmetric space, the condition is violated and $\mathcal{O}(N)$ entries of the Hessian matrix need to be tuned to ensure stability. However, even when the geometric condition is not satisfied, approximate no-scale dS vacua can be obtained with a moderate tuning, as we explicitly illustrate by constructing simple de Sitter solutions in STU supergravity.

While the realisations of this supersymmetry breaking scheme in the simplest models with a single no-scale field are somewhat restricted and do not allow for an exponentially large volume, we know of no reason why the general mechanism could not be applied

\footnotetext{
${ }^{2}$ Typical critical points in random supergravity for which the supersymmetry breaking scale of the same order as the supersymmetric masses, $m_{3 / 2} \approx m_{\text {susy }}$, the fraction of metastable critical points, $P$, scale with $N$ like $\ln P \sim-N^{2}$. For approximately supersymmetric critical points with $m_{3 / 2} \ll m_{\text {susy }}$, the corresponding fraction scales like $\ln P \sim-N$.
} 
to the case of multiple Kähler moduli in which an exponentially large volume might be achievable. Extending this scheme to more general sectors of no-scale fields is an important future direction.

This paper is organised as follows: in section 2, we review no-scale Minkowski vacua of $\mathcal{N}=1$ supergravity. We continue in section 3 to show how small deformations of the superpotential can perturb such no-scale vacua into metastable de Sitter vacua, with all scalars stabilised. In particular, we discuss the 'no-go' result that such perturbations are not possible unless the unperturbed no-scale vacuum has a third real massless direction. We also explain the generalities of the decoupling mechanism that ensures the stability of $2 N-3$ real modes of the Hessian, and we derive the surprisingly simple form of the spectrum of the remaining three modes. In section 4, we present realisations of the mechanism in supergravity and string theory, and discuss the generalities of models in which the noscale modulus appears in the superpotential through a single non-perturbative exponential term. We also consider the realisation of the mechanism in type IIB flux vacua, and derive a geometric condition on the complex structure field space geometry for minimal $\mathcal{O}(1)$ tuning. Finally, we discuss the realisation of the mechanism in STU supergravity models. We conclude in section 5, and list some useful formulae in appendix A.

\section{Unbroken no-scale vacua}

The remarkable properties of no-scale supergravities [30] have long been appreciated by many authors (for a colloquial review of some of the early developments, see for instance [31]). In this section, we review some elementary results on moduli stabilisation in unbroken no-scale vacua, and introduce some useful notation.

For the no-scale Kähler potential of equation (1.1) and the superpotential of equation (1.2), the scalar potential in equation (1.3) has no-scale solutions with $F_{T}=K_{T} W\left(X^{i}\right)$ and $F_{i}=0$. For these vacua, the potential (1.3) results in vanishing entries in the Hessian for all components involving $T$ or $\bar{T}$ :

$$
m_{T \bar{T}}^{2}=m_{T T}^{2}=m_{T i}^{2}=m_{\bar{T} i}^{2}=0,
$$

where we have adopted the notation $\partial_{a b}^{2} V=e^{K} m_{a b}^{2}$ for the entries of the Hessian matrix. This is of course directly related to the vacuum expectation value of $T$ not being fixed in the no-scale solution. The fields $X^{i}$ have non-vanishing entries of the Hessian that are given by (see appendix A for useful formulae),

$$
\begin{aligned}
& m_{i \bar{\jmath}}^{2}=Z_{i k} \bar{Z}_{\bar{\jmath}}^{k}+|W|^{2} K_{i \bar{\jmath}}, \\
& m_{i j}^{2}=2 Z_{i j} \bar{W}
\end{aligned}
$$

where we have introduced the Kähler invariant and diffeomorphism covariant symmetric tensor $Z_{i j}=D_{i} D_{j} W=\partial_{i} F_{j}+K_{i} F_{j}-\Gamma_{i j}^{k} F_{k}$, which for $F_{i}=0$ reduces to $Z_{i j}=\partial_{i} F_{j}$. For future reference, we note that in this notation, the critical point equations, $\partial_{a} V=0$, can be written as [32],

$$
Z_{a b} \bar{F}^{b}=2 \bar{W} F_{a},
$$

and imply that the no-scale vacuum enforces $Z_{T i}=0$ and $Z_{T T}=2 W K_{T T}$, for $W \neq 0$. 
The Hessian matrix (1.4) for the no-scale system is then given by,

$$
\begin{aligned}
\mathcal{H} & =e^{K}\left(\begin{array}{cc|cc}
m_{T \bar{T}}^{2} & \overrightarrow{0}^{T} & m_{T T}^{2} & \overrightarrow{0}^{T} \\
\overrightarrow{0} & m_{i \bar{\jmath}}^{2} & \overrightarrow{0} & m_{i j}^{2} \\
\hline m_{\overline{T T}}^{2} & \overrightarrow{0}^{T} & m_{\bar{T} T}^{2} & \overrightarrow{0}^{T} \\
\overrightarrow{0} & m_{\bar{\imath} \bar{\jmath}} & \overrightarrow{0} & m_{\bar{\imath} j}^{2}
\end{array}\right)= \\
= & e^{K}\left(\begin{array}{cc|cc}
0 & \overrightarrow{0}^{T} & 0 & \overrightarrow{0}^{T} \\
\overrightarrow{0} & Z_{i k} \bar{Z}^{k}+K_{i \bar{\jmath}}|W|^{2} & \overrightarrow{0} & 2 Z_{i j} \bar{W} \\
\hline 0 & \overrightarrow{0}^{T} & 0 & \overrightarrow{0}^{T} \\
\overrightarrow{0} & 2 \bar{Z}_{\bar{\imath} \bar{\jmath}} W & \overrightarrow{0} & \bar{Z}_{\bar{\imath} \bar{k}} Z^{\bar{k}}{ }_{j}+K_{\bar{\imath} j}|W|^{2}
\end{array}\right) .
\end{aligned}
$$

We note in particular that the 'off-diagonal' terms, $m_{i j}^{2}$ are not necessarily small compared to the 'diagonal block', $m_{i \bar{\jmath}}^{2}$. Since the potential (1.3) is semi-positive definite and vanishes at the no-scale minimum, the eigenvalues of the Hessian matrix must also be semi-positive definite. To see this explicitly, we diagonalise the Hessian matrix. For ease of representation we neglect the trivial $T$ and $\bar{T}$ directions and choose a basis in which the fields are canonically normalised at the critical point, $\left.K_{i \bar{j}}\right|_{\text {c.p. }}=\delta_{i \bar{\jmath}}$. The complex symmetric matrix $Z_{i j}$ can be Takagi factorized as $Z=U \Sigma U^{T}$, where $U$ is a unitary matrix whose columns are orthonormal eigenvectors of $Z \bar{Z}, \Sigma=\operatorname{diag}\left(\lambda_{1}, \ldots \lambda_{N-1}\right)$, and the $\lambda_{i}$ are real and nonnegative, with $\lambda_{i}^{2}$ the eigenvalues of $Z \bar{Z}$. We note that upon performing the $(2 N-2) \times(2 N-2)$ unitary transformation

$$
\mathcal{H} \rightarrow \mathcal{U}^{\dagger} \mathcal{H} \mathcal{U} \quad \text { with } \quad \mathcal{U}=\left(\begin{array}{cc}
U & 0 \\
0 & \bar{U}
\end{array}\right),
$$

the matrix $\mathcal{H}$ can be written as

$$
\mathcal{H}=\left(\begin{array}{cc}
\Sigma^{2}+\delta_{i \bar{\jmath}}|W|^{2} & 2 \bar{W} \Sigma \\
2 W \Sigma & \Sigma^{2}+\delta_{\bar{\imath} j}|W|^{2}
\end{array}\right) .
$$

After rearranging the rows and columns in an obvious way, $\mathcal{H}$ takes the block diagonal form

$$
\mathcal{H}=\left(\begin{array}{ccccc}
\lambda_{1}^{2}+|W|^{2} & 2 \bar{W} \lambda_{1} & 0 & 0 & \\
2 W \lambda_{1} & \lambda_{1}^{2}+|W|^{2} & 0 & 0 & \\
0 & 0 & \lambda_{2}^{2}+|W|^{2} & 2 \bar{W} \lambda_{2} & \\
0 & 0 & 2 W \lambda_{2} & \lambda_{2}^{2}+|W|^{2} & \\
& & & & \ddots
\end{array}\right) .
$$


Each $2 \times 2$ block can be diagonalised by the unitary transformation,

$$
u_{2 \times 2}=\frac{1}{\sqrt{2}}\left(\begin{array}{cc}
1 & 1 \\
e^{i \vartheta_{W}} & -e^{i \vartheta_{W}}
\end{array}\right),
$$

where $W=e^{i \vartheta_{W}}|W|$ and the corresponding eigenvalues of $\mathcal{H}$ are given by

$$
m_{i \pm}^{2}=\lambda_{i}^{2} \pm 2|W| \lambda_{i}+|W|^{2}=\left(\lambda_{i} \pm|W|\right)^{2},
$$

showing that the Hessian $\mathcal{H}$ is manifestly semi-positive definite.

In sum, no-scale vacua have $T$ unfixed with a vanishing mass, a vanishing cosmological constant and a semi-positive definite spectrum for the $X^{i}$ fields. In this paper, we investigate how small no-scale breaking perturbations to the superpotential, $\delta W\left(T, X^{i}\right)$, may lift the $T$ modulus and give rise to meta-stable vacua with a positive cosmological constant.

\section{Approximate no-scale vacua}

In this section, we discuss how small perturbations to the superpotential can result in potentials admitting metastable de Sitter vacua. The no-go theorem of [33] is then relevant: if the supersymmetry breaking $F$-term is completely aligned with a single field, $T$, with a no-scale Kähler potential, then no dS minima are obtainable, independently of the form of the superpotential. Here, we keep the unperturbed form of the Kähler potential, (1.1), and only consider superpotential corrections as

$$
W=W_{0}(X)+\delta W\left(T, X^{i}\right),
$$

which then necessarily have to induce non-vanishing $F$-terms for some of the fields perpendicular to $T$, as discussed in [28].

We are particularly interested in the regime in which the superpotential correction is small compared to $W_{0}$, such that $\left|\delta W / W_{0}\right| \ll 1$, and we furthermore assume that derivatives of $\delta W\left(T, X^{i}\right)$ are not very large compared to the scale of the perturbation itself. This can for instance be realised if $\delta W\left(T, X^{i}\right)$ is given by the sum of some non-perturbative corrections which are all small compared to $W_{0} \cdot{ }^{3}$ In natural units and denoting partial derivatives of the superpotential with subscripts, we then expect,

$$
\mathcal{O}(\delta W) \sim \mathcal{O}\left(\delta W_{T}\right) \sim \mathcal{O}\left(\delta W_{i}\right) \sim \mathcal{O}\left(\delta W_{T i}\right) \ll \mathcal{O}\left(W_{0}\right) \sim \mathcal{O}\left(W_{j}\right) \sim \mathcal{O}\left(W_{i j}\right) .
$$

This assumed hierarchy for the superpotential terms justifies a perturbative expansion in the no-scale breaking.

For the approximate no-scale solution, we may write the $F$-terms at the minimum as,

$$
\begin{aligned}
F_{T} & =K_{T} W+\delta W_{T}, \\
F_{i} & =\epsilon W f_{i},
\end{aligned}
$$

where $f_{i}$ is a unit vector, $\left\|f_{i}\right\|=1$, and we study the perturbative expansion in the small parameter $\epsilon$.

\footnotetext{
${ }^{3}$ We do note however that these conditions are not satisfied in some classes of string compactifications: for example, in KKLT vacua $\left|W_{0}\right|$ is tuned to be small compared to the flux scale and $\left|\delta W / W_{0}\right|$ is not small.
} 


\subsection{Critical point equations}

The critical point equations in the $T$ and $X^{i}$ directions determine much of the structure of the approximately no-scale vacua. The equation $\partial_{T} V=0$ is to leading order in a perturbative expansion in $\epsilon$ and $\left|\delta W / W_{0}\right|$ given by

$$
K_{T T}\left|W_{0}\right|^{2} \epsilon^{2}=-\bar{W}_{0} \delta W_{T T}-\frac{4}{3} K_{T} \operatorname{Re}\left(\bar{W}_{0} \delta W_{T}\right) .
$$

Two properties of this equation are particularly noteworthy: first, while the $F$-term in the $X^{i}$ subspace is corrected at $\mathcal{O}(\epsilon)$, derivatives of $\delta W$ - which we by equation (3.2) relate to the magnitude of $|\delta W|$ - appear first at order $\epsilon^{2}$. Thus, consistently with equation (3.2) we can realise the scaling,

$$
\mathcal{O}\left(\epsilon^{2} W_{0}\right) \sim \mathcal{O}(\delta W) \sim \mathcal{O}\left(\delta W_{T}\right) \sim \mathcal{O}\left(\delta W_{T i}\right) \ll \mathcal{O}\left(W_{0}\right) \sim \mathcal{O}\left(W_{j}\right) \sim \mathcal{O}\left(W_{i j}\right)
$$

Equation (3.5) then receives subleading corrections at $\mathcal{O}\left(\epsilon^{3}\right)$, and the smallness of the Fterms in the $X^{i}$ subspace assumed in equation (3.4) may be achieved by the tuning of $W_{0}\left(X^{i}\right)$. Second, the reality of the left-hand side of equation (3.5) enforces that,

$$
\operatorname{Im}\left(\bar{W}_{0} \delta W_{T T}\right)=0
$$

The remaining critical point equation, $\partial_{i} V=0$, is most illuminatingly phrased as a condition on the supersymmetric masses of the $X^{i}$ fields, explicitly given by an eigenvalue equation involving $Z_{i j}$. To see this, we note that to leading order,

$$
Z_{T i}=\delta W_{T i}+K_{i} \delta W_{T}+K_{T} F_{i}=K_{T} F_{i}+\mathcal{O}\left(\epsilon^{2}\right),
$$

so that the critical point equation $\partial_{i} V=0$, which can also be written as $Z_{i a} \bar{F}^{a}=2 \bar{W} F_{i}$ as in equation (2.4), implies that,

$$
Z_{i j} \bar{F}^{j}=-\bar{W} F_{i}
$$

up to corrections of $\mathcal{O}\left(\epsilon^{2}\right)$. Contracting the complex conjugate of the equation above with $Z_{i}{ }^{\bar{j}}$ and using the equation again, we find to linear order in $\epsilon$,

$$
(Z \bar{Z})_{i}^{j} F_{j}=|W|^{2} F_{i}
$$

implying that $Z \bar{Z}$ has an eigenvalue equal to $|W|^{2}$ to this order (in the basis in which $\left.K_{i \bar{\jmath}}=\delta_{i \bar{\jmath}}\right)$, with $F_{i}$ being the corresponding eigenvector.

Equation (3.10) directly affects the spectrum of approximately no-scale vacua. From equation (2.10) we find that it implies the existence of one real field in the $X^{i}$-sector with a vanishing mass at zeroth order in $\epsilon$. Thus, out of all exact no-scale vacua, only the subset with at least three real massless degrees of freedom can be perturbatively lifted to de Sitter vacua (or deformed to non-supersymmetric AdS vacua).

The interpretation of the critical point equation as a condition on the supersymmetric mass spectrum is quite familiar: in [34] it was shown that for critical points at which supersymmetry is spontaneously broken in the $X^{i}$-sector, without a dominant contribution from 
the no-scale field, obey a similar eigenvalue equation derived from equation (2.4). Consistently, supersymmetric Minkowski solutions that can be perturbed to non-supersymmetric vacua also have at least one massless direction [28].

Finally, upon using the critical point equation (3.5), we find that the vacuum expectation value of the scalar potential at the critical point is given by,

$$
\begin{aligned}
e^{-K} V & =2 \operatorname{Re}\left(K^{T} \bar{W}_{0} \delta W_{T}\right)+\left|F_{i}\right|^{2}+\mathcal{O}\left(\epsilon^{4}\right) \\
& =\frac{2}{3} K^{T} \operatorname{Re}\left(\bar{W}_{0} \delta W_{T}\right)-K^{T T} \bar{W}_{0} \delta W_{T T}+\mathcal{O}\left(\epsilon^{3}\right),
\end{aligned}
$$

where the reality of the scalar potential is ensured by equation (3.7).

\subsection{The perturbed mass-matrix}

By introducing a small amount of supersymmetry breaking in the directions perpendicular to $T$, we avoid the no-go theorem of [33] and may potentially find metastable de Sitter vacua. Since the additional supersymmetry breaking is small by assumption, a perturbative expansion around the no-scale vacuum is well-motivated. We now show that the Hessian matrix takes a very simple form upon performing such an expansion.

First, we note that the general expression for perturbed non-degenerate eigenvalues up to second order is given by,

$$
\left.m_{A}^{2}\right|_{\text {tot }}=\left.m_{A}^{2}\right|_{0}+\delta m_{A}^{2}+\sum_{B \neq A} \frac{\left|\delta m_{A B}^{2}\right|^{2}}{\left.m_{A}^{2}\right|_{0}-\left.m_{B}^{2}\right|_{0}}+\ldots
$$

This expression is not directly applicable to the perturbative analysis of the approximate no-scale vacua as three real degrees of freedom $(\operatorname{Re}(T), \operatorname{Im}(T)$ and one real component of the eigenstate of $Z \bar{Z}$ with eigenvalue $\left|W_{0}\right|^{2}$ ) are degenerate and massless at zeroth order in no-scale breaking. Taking this degeneracy into account, we will find equation (3.12) useful in determining the structure of the dominant contributions of the Hessian matrix. In the following section we describe how $2 N-3$ fields are stabilised with positive definite masses $\gtrsim \mathcal{O}\left(m_{3 / 2}\right)$, one real direction generically is lifted at $\mathcal{O}(\epsilon)$, and the Hessian eigenvalues of the remaining two fields take a strikingly simple form.

\subsubsection{Masses for the $X^{i}$ fields}

We begin by considering the spectrum of all fields perpendicular to $T$.

While one real direction in the $X^{i}$ field space has a vanishing mass at zeroth order (by equations (3.10) and (2.10), as discussed above), the remaining $X^{i}$ fields are expected to obtain masses of order $\max \left(\lambda_{i}, W_{0}\right)$. The no-scale breaking induces small perturbations to the exact spectrum of these fields, but for $\epsilon \ll 1$, the corrections are too small to destabilise these fields. This partial decoupling - which is a direct consequence of the noscale structure of the Kähler potential - significantly simplifies the problem of assessing the stability of the perturbed critical point. In principle, these $2 N-3$ real degrees of freedom may still affect the general stability of the system by Hessian cross-couplings that may destabilise lighter fields. We will see however, that the no-scale structure again makes these contributions negligible, thus ensuring the complete decoupling of all but one $X^{i}$ field. 
We now turn to the remaining, light $X^{i}$ field and determine its mass and couplings to leading order in the perturbative expansion of equation (3.6). These expressions are most easily analysed by considering the sub-matrix of the Hessian that involve only the $X^{i}$ fields, and in a basis in which the fields at the critical point are canonically normalised, i.e. $\left.K_{i \bar{\jmath}}\right|_{\text {c.p. }}=\delta_{i \bar{\jmath}}$. We will see in section 3.2.2 that this explicit neglect of the cross-terms between the $X^{i}$ fields and the no-scale modulus is perfectly justifiable to $\mathcal{O}\left(\epsilon^{2}\right)$.

The Hessian submatrix involving only the $X^{i}$ fields is to linear order in $\epsilon$ given by,

$$
\left(\begin{array}{c}
m_{i \bar{\jmath}}^{2} m_{i j}^{2} \\
m_{\bar{\imath} \bar{\jmath}}^{2} m_{\bar{\jmath} j}^{2}
\end{array}\right)=\left(\begin{array}{cc}
Z_{i k} \bar{Z}_{\bar{\jmath}}^{k}+\delta_{i \bar{\jmath}}|W|^{2} & 2 Z_{i j} \bar{W} \\
2 \bar{Z}_{\bar{\imath} \bar{\jmath}} W & \bar{Z}_{\bar{\imath} \bar{k}} Z^{\bar{k}}{ }_{j}+\delta_{\bar{\imath} j}|W|^{2}
\end{array}\right)+\left(\begin{array}{cc}
0 & \left(D_{i} Z_{j k}\right) \bar{F}^{k} \\
\left(\bar{D}_{\bar{\imath}} \bar{Z}_{\bar{\jmath} \bar{k}}\right) F^{\bar{k}} & 0
\end{array}\right) .
$$

Thus, in comparison with the no-scale Hessian of the $X^{i}$ field sector of equation (2.5), we note that the only structural correction to this sub-Hessian at $\mathcal{O}(\epsilon)$ is given by the second term of equation (3.13). We are interested in the lightest $X^{i}$ field, which - as discussed in section 3.1 - to linear order in $\epsilon$ corresponds to the eigenmode of $Z \bar{Z}$ with an eigenvector proportional to $F_{i}$. The corresponding orthonormalised (sub-)Hessian eigenvectors for the modes with eigenvalues 0 and $4|W|^{2}$ are given by,

$$
v_{1 \pm}=\frac{1}{\sqrt{2}}\left(\begin{array}{c}
f^{\bar{\imath}} \\
\mp \bar{f}^{i}
\end{array}\right)
$$

where we, as in equation (3.4), have introduced the unit vector $f_{i}=\exp \left(-i \vartheta_{W}\right) F_{i} /\left\|F_{i}\right\|$, where again $\vartheta_{W}=\arg (W)$.

We now note that Hessian cross-terms between the $v_{1-}$ direction and the more massive $X^{i}$ fields - that generically are lifted at $\mathcal{O}\left(\epsilon^{0}\right)$ - enter the mass matrix at $\mathcal{O}(\epsilon)$, as $v_{1 \pm}$ are eigenvectors of the Hessian to leading order. Thus, from equation (3.12), we see that they affect the mass of the lightest $X^{i}$ field at second order in perturbation theory by $\mathcal{O}\left(\epsilon^{2}\right) \ll$ $\mathcal{O}(\epsilon)$, and may consistently be neglected. This is a crucial property of the metastable vacua with only $\mathcal{O}(1)$ fine tuning that we construct in this paper.

The Hessian eigenvalues of the lightest $X^{i}$ field, which we will refer to as the ' 1 ' direction, are to linear order in $\epsilon$ then given by,

$$
\begin{aligned}
& m_{1+}^{2}=4|W|^{2}-\operatorname{Re}\left(\left(D_{i} Z_{j k}\right) \bar{f}^{i} \bar{f}^{j} \bar{f}^{k} \bar{W}\right) \epsilon, \\
& m_{1-}^{2}=\operatorname{Re}\left(\left(D_{i} Z_{j k}\right) \bar{f}^{i} \bar{f}^{j} \bar{f}^{k} \bar{W}\right) \epsilon .
\end{aligned}
$$

As a final word of caution, we note that if $\operatorname{Re}\left(\left(D_{i} Z_{j k}\right) \bar{f}^{i} \bar{f}^{j} \bar{f}^{k} \bar{W}\right)$ is of order $\mathcal{O}(\epsilon)$, then the structure of the $X^{i}$ sector sub-Hessian is identical to that of the unbroken no-scale Hessian to $\mathcal{O}(\epsilon)$, and the critical point equation (3.9) again enforces that the mass of the lightest field vanishes. Consequently, in this case the lightest $X^{i}$ field is lifted at $\mathcal{O}\left(\epsilon^{2}\right)$, and cross-couplings with heavier $X^{i}$ fields can then not be neglected in general. Metastability then requires that $\mathcal{O}(N)$ elements of the Hessian matrix may have to be tuned small.

In sum, most of the fields perpendicular to the no-scale field obtain positive definite masses of the order of $\max \left(\lambda_{i}, W_{0}\right)$ (cf. equation (2.10)) and do not develop instabilities in 
the approximately de Sitter no-scale vacuum. The lightest $X^{i}$ field is generically lifted at $\mathcal{O}(\epsilon)$, and the detailed form of the squared mass depends on the covariant tensor $D_{a} Z_{b c}$. It thus seems plausible that only a very modest amount of tuning of the superpotential parameters can result in positive eigenvalues for all the $X^{i}$ fields. In section 4 , we demonstrate that this is indeed true in explicit examples.

\subsubsection{Decoupling and stabilisation of the no-scale direction}

To find a metastable de Sitter minimum, we must also stabilise the no-scale modulus $T$. Upon using the critical point equations (3.5) and (3.9) we find that to quadratic order in $\epsilon$,

$$
\begin{aligned}
& m_{T \bar{T}}^{2}=-\frac{4}{3} K_{T} \operatorname{Re}\left(W \delta \bar{W}_{\bar{T}}\right) \\
& m_{T T}^{2}=K^{T} \bar{W} \delta W_{T T T}-\frac{4}{3} K_{T} \operatorname{Re}\left(\bar{W} \delta W_{T}\right)
\end{aligned}
$$

Since these contributions to the mass of the no-scale modulus are of order $\mathcal{O}\left(\epsilon^{2}\right)$, and thus quite small compared to other entries of the Hessian matrix, some care must be taken to ensure that cross-couplings do not destabilise the no-scale field. Naively, this would require the fine-tuning of the $2(N-1)$ elements, $m_{T i}^{2}, m_{\bar{T} i}^{2}$ of the Hessian, however, we now show that such fine-tuning is not necessary: enforcing the critical point equations ensures that none of the cross-terms contribute with any significant destabilising terms.

In section 2 we showed that $m_{i T}^{2}$ and $m_{i \bar{T}}^{2}$ vanish in the no-scale vacuum. The correction at $\mathcal{O}(\epsilon)$ is given by,

$$
\begin{aligned}
& m_{i \bar{T}}^{2}=K_{\bar{T}}\left(Z_{i j} \bar{F}^{j}+\bar{W} F_{i}\right)+\mathcal{O}\left(\epsilon^{2}\right)=\mathcal{O}\left(\epsilon^{2}\right), \\
& m_{i T}^{2}=K_{T}\left(Z_{i j} \bar{F}^{j}+\bar{W} F_{i}\right)+\mathcal{O}\left(\epsilon^{2}\right)=\mathcal{O}\left(\epsilon^{2}\right),
\end{aligned}
$$

where we used the critical point equation (3.9) in the last step of both equations.

In second order perturbation theory, these terms then contribute negatively to the eigenvalues of the (mostly) $T$-field eigenstates at order $\mathcal{O}\left(\epsilon^{4} W_{0}^{4} / m_{i \pm}^{2}\right)$ according to equation (3.12). Thus, the contribution from the lightest $X^{i}$ field is expected to enter at $\mathcal{O}\left(\epsilon^{3}\right)$, while the other $X^{i}$ fields contribute at order $\mathcal{O}\left(\epsilon^{4}\right)$. However, as the leading contribution from $m_{T T}^{2}$ and $m_{T \bar{T}}^{2}$ enter at $\mathcal{O}\left(\epsilon^{2}\right)$, these potentially destabilising corrections are subleading and do not contribute significantly to the (de-)stabilisation of $T$ (as long as $N \lesssim 1 / \epsilon^{2}$ ). Indeed, the smallest eigenvalues of the Hessian matrix are to $\mathcal{O}\left(\epsilon^{2}\right)$ given by,

$$
\begin{aligned}
m_{T \pm}^{2} & =m_{T \bar{T}}^{2} \pm\left|m_{T T}^{2}\right| \\
& =-\frac{4}{3} K_{T} \operatorname{Re}\left(\bar{W} \delta W_{T}\right) \pm\left|K^{T} \bar{W} \delta W_{T T T}-\frac{4}{3} K_{T} \operatorname{Re}\left(\bar{W} \delta W_{T}\right)\right|
\end{aligned}
$$

Equation (3.21) is one of our main results, and indicates that — given some small amount of supersymmetry breaking in the directions perpendicular to $T$ - all moduli can be stabilised in approximate no-scale compactifications with a very small amount of tuning. 
Let us summarise: the Hessian matrix of the approximately no-scale system is schematically given by,

$$
\begin{aligned}
& \mathcal{H}=e^{K}\left(\begin{array}{c|c|c|c|c|c}
m_{T \bar{T}}^{2} & m_{T \overline{1}}^{2} & m_{T \bar{\jmath}^{\prime}}^{2} & m_{T T}^{2} & m_{T 1}^{2} & m_{T j^{\prime}}^{2} \\
\hline m_{1 \bar{T}}^{2} & m_{1 \overline{1}}^{2} & m_{1 \bar{\jmath}^{\prime}}^{2} & m_{1 T}^{2} & m_{11}^{2} & m_{1 j^{\prime}}^{2} \\
\hline m_{i^{\prime} \bar{T}}^{2} & m_{i^{\prime} \overline{1}}^{2} & m_{i^{\prime} \bar{\jmath}^{\prime}}^{2} & m_{i^{\prime} T}^{2} & m_{i^{\prime} 1}^{2} & m_{i^{\prime} j^{\prime}}^{2} \\
\hline m_{\bar{T} T}^{2} & m_{\bar{T} \overline{1}}^{2} & m_{\bar{T} \bar{\jmath}^{\prime}}^{2} & m_{\bar{T} T}^{2} & m_{\bar{T} 1}^{2} & m_{\bar{T} j^{\prime}}^{2} \\
\hline m_{\overline{1} \bar{T}}^{2} & m_{\overline{1} \overline{1}}^{2} & m_{\overline{1} \bar{\jmath}^{\prime}}^{2} & m_{\overline{1} T}^{2} & m_{\overline{1} 1}^{2} & m_{\overline{1} j^{\prime}}^{2} \\
\hline m_{\bar{\imath}^{\prime} \bar{T}}^{2} & m_{\bar{\imath}^{\prime} \overline{1}}^{2} & m_{\bar{\imath}^{\prime} \bar{\jmath}^{\prime}}^{2} & m_{\bar{\imath}^{\prime} T}^{2} & m_{\bar{\imath}^{\prime} 1}^{2} & m_{\bar{\imath}^{\prime} j^{\prime}}^{2}
\end{array}\right)= \\
& =m_{3 / 2}^{2}\left(\begin{array}{c|c|c|c|c|c}
\sim \epsilon^{2} & \sim \epsilon^{2} & \sim \epsilon^{2} & \sim \epsilon^{2} & \sim \epsilon^{2} & \sim \epsilon^{2} \\
\hline \sim \epsilon^{2} & \sim \epsilon & \sim \epsilon & \sim \epsilon^{2} & \sim \epsilon & \sim \epsilon \\
\hline \sim \epsilon^{2} & \sim \epsilon & \sim 1 & \sim \epsilon^{2} & \sim \epsilon & \sim 1 \\
\hline \sim \epsilon^{2} & \sim \epsilon^{2} & \sim \epsilon^{2} & \sim \epsilon^{2} & \sim \epsilon^{2} & \sim \epsilon^{2} \\
\hline \sim \epsilon^{2} & \sim \epsilon & \sim \epsilon & \sim \epsilon^{2} & \sim \epsilon & \sim \epsilon \\
\hline \sim \epsilon^{2} & \sim \epsilon & \sim 1 & \sim \epsilon^{2} & \sim \epsilon & \sim 1
\end{array}\right),
\end{aligned}
$$

where we for simplicity of presentation have taken $\mathcal{O}\left(\lambda_{a}\right) \sim \mathcal{O}\left(\left|W_{0}\right|\right)$ to set the scale of the matrix, and highlighted the dominant elements.

We have shown that most $X^{i}$ fields decouple at zeroth order in the no-scale breaking and receive squared masses of the order of $\mathcal{O}\left(\epsilon^{0}\right)$, cf. equation (2.10). The critical point equations imply that only no-scale systems with three real flat directions at zeroth order in the no-scale breaking support approximately de Sitter solutions, as discussed in section 3.1.

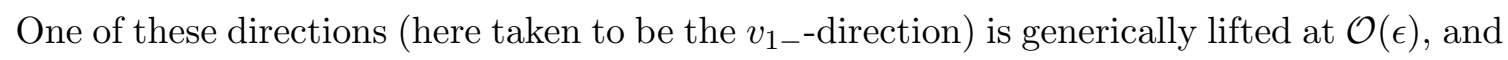
the corresponding squared mass may be rendered positive by a modest amount of tuning. The remaining two flat directions are lifted at $\mathcal{O}\left(\epsilon^{2}\right)$, with no significant contribution coming from the cross-terms with other moduli. 
Thus, the approximately no-scale de Sitter vacua are meta-stable if $m_{1-}^{2}>0$, which corresponds to

$$
\operatorname{Re}\left(\left(D_{i} Z_{j k}\right) \bar{f}^{i} \bar{f}^{j} \bar{f}^{k} \bar{W}\right)>0,
$$

and $m_{T-}^{2}=m_{T \bar{T}}^{2}-\left|m_{T T}^{2}\right|>0$, as given by equation (3.21). The tuning of the Hessian matrix necessary to obtain such vacua is of order $\mathcal{O}(1)$, which should be compared with $\mathcal{O}\left(N^{2}\right)$ for similar vacua obtained in [28].

\section{Examples in supergravity and string theory}

We expect that the general properties of the approximately no-scale vacua considered in this paper are broadly applicable to many different examples, including those involving more complicated, multi-field no-scale Kähler potentials. Here, we will as a proof of principle demonstrate that this type of vacua is indeed obtainable in supergravity, and in particular we focus on string theory inspired supergravities of STU-type. In the simplest scenarios, we find some limiting conditions. For more involved examples, we expect these constraints to be relaxed, and we look forward to exploring these possibilities in future work.

Before delving into the details of an explicit STU-model, we first note some common properties of large classes of models.

\subsection{Models with one non-perturbative effect: generalities}

The simplest model one can consider which realises decoupling from approximate no-scale vacua involves a single non-perturbative, no-scale breaking superpotential correction,

$$
\delta W=A\left(X^{i}\right) e^{-a T} .
$$

By performing a trivial, constant Kähler transformation, we can choose $W_{0} \in \mathbb{R}_{+}$at the critical point. The stabilisation of the $T$-axion is ensured by equation (3.7), from which we infer that at the critical point,

$$
\delta W=s|\delta W|=s|A| e^{-a \tau}, \quad \tau=\operatorname{Re} T,
$$

where $\tau$ denotes the real part of $T$ and $s \in\{-1,+1\}$. From equation (3.17) we see that $s=+1$ corresponds to $m_{T \bar{T}}<0$, which immediately implies that there is a tachyon. We therefore set $s=-1$. The value of the scalar potential at the critical point is given by equation (3.11), which evaluates to

$$
V=e^{K} K^{T T} a^{2} W_{0}|\delta W|\left(1-\frac{1}{a \tau}\right) .
$$

Thus, minima with $V>0$ have $a \tau>1$. The smallest scalar mass is easily computed from equation (3.21)

$$
m_{T-}^{2}=m_{T \bar{T}}^{2}-\left|m_{T T}^{2}\right|=\frac{2 a}{\tau} W_{0}|\delta W|\left(1-\left|1-\tau^{2} a^{2}\right|\right) .
$$

Thus, we find that $m_{T-}^{2}>0$ for $a \tau<\sqrt{2}$. In sum, metastable de Sitter can be found only for

$$
1<a \tau<\sqrt{2} .
$$


The above constraint (4.5) appears very restrictive and one might wonder whether it can be realised in a controlled supergravity regime. Since we clearly want $\tau=\operatorname{Re}(T) \gg 1$ to suppress $\alpha^{\prime}$ corrections we need $a \ll 1$. In type IIB string theory this may be achievable if the non-perturbative term (4.1) arises from gaugino condensation on a large stack of D7-branes. In this case the full correction has the form $\delta W=A e^{-a f\left(T, X^{i}\right)}$ where $f\left(T, X^{i}\right)$ is the gauge kinetic function [35]. Due to the non-renormalization theorem of [36], the gauge kinetic function takes the form $f\left(T, X^{i}\right)=f^{\text {tree }}(T)+f^{1-\operatorname{loop}}\left(X^{i}\right)+f^{\mathrm{np}}\left(T, X^{i}\right)=T+f^{1-\operatorname{loop}}\left(X^{i}\right)+$ $\sum_{n=1}^{\infty} c_{n}\left(X^{i}\right) e^{-a n T}$. For $\operatorname{Re}(T) \gg 1$, it seems reasonable to assume that $f\left(T, X^{i}\right) \approx$ $f^{\text {tree }}(T)=T$, but it would certainly be interesting to check this in explicit models.

Furthermore, the critical point equation (3.5) implies to leading order in $\epsilon$ that,

$$
W_{0}^{2} \epsilon^{2}=\frac{4}{3} W_{0}|A| f(a \tau),
$$

where we have defined $f(x)=\left(x^{2}+2 x\right) \exp (-x)$. The function $f(x)$ is semi-monotonically increasing in the range $x \in[1, \sqrt{2}]$, and reaches a maximum at $x=\sqrt{2}$. As $f(x)$ is bounded, this constrains the ratio between $\epsilon^{2}$ and $|A| / W_{0}$ obtainable in any model. In particular we find

$$
\frac{3}{4} \frac{\epsilon^{2}}{f_{\max }}<\frac{|A|}{W_{0}}<\frac{3}{4} \frac{\epsilon^{2}}{f_{\min }}
$$

where $f_{\min }=f(1)=3 / e \approx 1.104$ and $f_{\max }=f(\sqrt{2})=2(1+\sqrt{2}) \exp (-\sqrt{2}) \approx 1.174$. Numerically, we then have,

$$
0.639<\frac{1}{\epsilon^{2}} \frac{|A|}{W_{0}}<0.680 .
$$

Thus any realisation of de Sitter vacua through the mechanism we have described above including the exact no-scale Kähler potential for a single no-scale field and a single nonperturbative term is somewhat constrained. As we have seen, the constraint (4.8) follows directly from the critical equation $\partial_{T} V=0$, which requires $\delta W_{T}=O\left(\epsilon^{2}\right)$, and the metastability condition $a \tau<\sqrt{2}$ from equation (4.5). Adding more Kähler fields could in principle allow for less restricted models, and it would be interesting to study this in detail.

\subsection{Simple examples in supergravity}

In this section, we illustrate the general mechanism of decoupling by an approximate noscale structure in the simplest possible supergravity models. While we do not expect these to directly capture the intricacies of supergravities arising from the dimensional reduction of string theory, they nicely illustrate the basic properties of the general mechanism.

First, we consider a system of two chiral superfields, $T$ and $X$ with the Kähler potential,

$$
K=-3 \ln (T+\bar{T})+X \bar{X}
$$

and the superpotential $W=W_{0}(X)+W_{n p}(T)$ with,

$$
\begin{aligned}
W_{0} & =w_{0}+\epsilon w_{0} X-\frac{w_{0}}{2} X^{2}+\frac{w_{3}}{3 !} X^{3}, \\
W_{n p} & =A e^{-a T},
\end{aligned}
$$


where we take $w_{0}, w_{3} \in \mathbb{R}$. For $\epsilon \ll 1$, this system has a non-supersymmetric solution at $X=0$ with $F_{X}=\epsilon w_{0}$ and $F_{T}=K_{T} w_{0}+\mathcal{O}\left(W_{n p}\right)$. Consistently with equations (2.10) and (3.10), the real and imaginary components of $X$ have masses which to zeroth order in $\epsilon$ are given by $m_{X+}^{2}=4 w_{0}^{2}$, and $m_{X-}^{2}=0$. At linear order in $\epsilon$ we find that,

$$
\begin{aligned}
& m_{X \bar{X}}^{2}=2 w_{0}^{2}+\mathcal{O}\left(\epsilon^{2}\right), \\
& m_{X X}^{2}=-2 w_{0}^{2}+\epsilon w_{0} w_{3}+\mathcal{O}\left(\epsilon^{2}\right) .
\end{aligned}
$$

The lightest component of the $X$ field is then metastable for $0<\epsilon w_{0} w_{3}<4 w_{0}^{2}$, and the stabilisation of the no-scale modulus may proceed as in section 4.1. Thus, we conclude that the mechanism presented in this paper admits very simple explicit realisations.

The decoupling mechanism discussed in this paper is readily generalised to systems with many interacting fields, and can, in particular be realised in 'random supergravity' [29]. To illustrate this, we consider an ensemble of critical points in which the value of the superpotential and the tensors $Z_{i j}$ and $D_{i} Z_{j k}$ are taken to be random tensors with independent and identically distributed entries, subject to the critical point equations (3.5) and (3.9) . The no-scale sector involving $T$ is not assumed to be random, but rather to be stabilised as in the discussion of section 4.1, or generalisations thereof. Consistently with our general discussion, simulations of such systems with $\mathcal{O}(100)$ fields show that most of the $X^{i}$ fields are stabilised with masses $m \gtrsim m_{3 / 2}$, and a single real field in the $X^{i}$-sector remains significantly lighter than $m_{3 / 2}$. The fraction of all critical points that are metastable vacua is $P=1 / 2$, independently of $N$, in agreement with our discussion around equation (3.16). This should be compared with the corresponding fractions for critical points without an underlying no-scale structure $[29,37,38]$ : for typical critical points with $\langle W\rangle^{2} \approx \mathcal{O}(\operatorname{eig}(Z \bar{Z}))$, a fraction of $P \approx \exp \left(-0.2 N^{2}\right)$ of the typical critical points with are metastable, and for approximately supersymmetric critical points with $\langle W\rangle^{2} \ll \mathcal{O}(\operatorname{eig}(Z \bar{Z}))$, the metastable fraction is given by $P \approx \exp (-0.35 N)$. Thus, in random supergravity, the relative frequency, $P$, of metastable approximately no-scale vacua is exponentially larger than the corresponding value for vacua without this structure.

\subsection{Examples in type IIB string theory: generalities}

An appealing feature of the class of de Sitter vacua presented in this paper is that they rely on ingredients which are readily available in compactification of string theory on CalabiYau manifolds. In particular, flux compactifications of type IIB string theory include all the ingredients necessary to realise this scenario: the Kähler potential for the Kähler moduli sector is of the no-scale form to leading order in the $\alpha^{\prime}$ and $g_{s}$ expansions, and it is plausible that among the possible choices of quantised three-form flux, there are theories admitting $\epsilon \ll 1$. In this section, we consider such flux vacua in more detail, and derive a geometric condition on the complex structure moduli space which must be satisfied in order to achieve de Sitter vacua with an $\mathcal{O}(1)$ fine-tuning of the Hessian matrix.

Type IIB string theory compactified on an orientifold, $\tilde{M}_{3}$, of a Calabi-Yau threefold $M_{3}$, reduces to a four-dimensional $\mathcal{N}=1$ effective supergravity if the supersymmetry breaking scale is much smaller than the compactification scale. The relevant degrees of 
freedom of such compactifications include the axio-dilaton, $S=e^{-\phi}-i C_{0}$, the complex structure moduli, $U^{i}$, where $i=1, \ldots, h_{-}^{2,1}\left(\tilde{M}_{3}\right)$, the complexified Kähler moduli, $T^{r}$, where $r=1, \ldots, h_{+}^{1,1}\left(\tilde{M}_{3}\right)$, the 2 -form axion multiplets $G^{\alpha}$ with $\alpha=1, \ldots, h_{-}^{1,1}\left(\tilde{M}_{3}\right)$ and possibly open string moduli. Many simple orientifolds have $h_{-}^{1,1}\left(\tilde{M}_{3}\right)=0$ and therefore no scalars arising from the $B_{2}$ and $C_{2}$ fields. For simplicity, we here consider only this case, and we furthermore do not explicitly include the action of additional open string degrees of freedom arising from stacks of D-branes.

The leading order Kähler potential for the Kähler moduli is given by $K_{\text {no-scale }}=$ $-2 \ln \mathcal{V}$, where $\mathcal{V}$ denotes the volume of the compactification manifold in units of $\alpha^{\prime}$. In this paper, we focus on models with a single Kähler modulus, $T$, and as $\mathcal{V} \sim(T+\bar{T})^{3 / 2}$, the Kähler modulus may then play the role of the no-scale field of equation (1.1).

The Kähler potential for the complex structure moduli, $U^{i}$, and the axio-dilaton, $S$, is given by

$$
\tilde{K}\left(S, \bar{S}, U^{i}, \bar{U}^{\bar{\imath}}\right)=-\ln (S+\bar{S})-\ln \left(i \int_{\tilde{M}_{3}} \Omega \wedge \bar{\Omega}\right),
$$

where $\Omega$ denotes the holomorphic (3,0)-form on $\tilde{M}_{3}$. Thus, to leading order in $\alpha^{\prime}$ and $g_{s}$, and in the absence of open string moduli, these fields do not mix with $T$ in the Kähler potential and may serve as our $X^{i}$ field sector.

We discuss the superpotential and its Kähler covariant derivatives, for more details see for instance [32]. Compactifications with quantised NS-NS flux, $H_{3}=d B_{2}$, and RR flux, $F_{3}=d C_{2}$, on non-trivial three-cycles of $\tilde{M}_{3}$ have a superpotential which is a linear combination of the periods of $\Omega$,

$$
W=\int_{\tilde{M}_{3}} \Omega \wedge G_{3}=\vec{N} \cdot \vec{\Pi},
$$

where we have introduced the complex three-form flux $G_{3}=F_{3}-i S H_{3}$, which takes on $2\left(h^{2,1}+1\right)$ quantised values as $\vec{N}=\vec{f}-i S \vec{h}$. Here $\vec{\Pi}$ denotes the period vector of the three-form $\Omega$.

The $F$-terms in the axio-dilaton and complex structure sector are then given by

$$
\begin{gathered}
F_{S}=D_{S} W=-\frac{\vec{N}^{*} \cdot \vec{\Pi}}{S+\bar{S}}, \\
F_{i}=D_{i} W=\vec{N} \cdot D_{i} \vec{\Pi} .
\end{gathered}
$$

The tensor $Z_{a b}$ has the components,

$$
\begin{aligned}
Z_{S S} & =0, \\
Z_{S i} & =-\frac{\vec{N}^{*} \cdot D_{i} \vec{\Pi}}{S+\bar{S}}, \\
Z_{i j} & =\mathcal{F}_{i j k} \vec{N} \cdot \bar{D}^{k} \vec{\Pi}^{*}=-(S+\bar{S}) \mathcal{F}_{i j k} \bar{Z}_{\bar{S}}^{k},
\end{aligned}
$$

where we have used the identity $D_{i} D_{j} \Omega=\mathcal{F}_{i j k} \bar{D}^{k} \bar{\Omega}$, with $\mathcal{F}_{i j k}$ denoting the 'Yukawa couplings' of the special geometry. ${ }^{4}$

\footnotetext{
${ }^{4}$ The coefficients $\mathcal{F}_{i j k}$ can be obtained by taking three holomorphic derivatives of the $\mathcal{N}=2$ prepotential, or by evaluating $\mathcal{F}_{i j k}=i \int \Omega(U) \wedge \frac{\partial^{3} \Omega(U)}{\partial U^{i} \partial U^{j} \partial U^{k}}=i \int \Omega(U) \wedge D_{i} D_{j} D_{k} \Omega(U)$. These 'Yukawa couplings' determine the field space Riemann curvature as, $R_{i \bar{\jmath} k \bar{\ell}}=-K_{i \bar{\jmath}} K_{k \bar{\ell}}-K_{i \bar{\ell}} K_{k \bar{\jmath}}+\exp (K) \mathcal{F}_{i k m} \overline{\mathcal{F}}_{\bar{\jmath} \bar{\ell}}{ }^{m}$.
} 
Furthermore, upon acting with three covariant derivatives on the superpotential, we find the components,

$$
\begin{aligned}
D_{S} Z_{S S} & =0, \quad D_{i} Z_{S S}=0 \\
D_{i} Z_{j S} & =-\frac{\mathcal{F}_{i j k} \bar{F}^{k}}{S+\bar{S}} \\
D_{i} Z_{j k} & =\mathcal{F}_{i j k} \vec{N} \cdot \vec{\Pi}^{*}+D_{k}\left(\mathcal{F}_{i j \ell}\right) \vec{N} \cdot \bar{D}^{\ell} \vec{\Pi}^{*}= \\
& =-\mathcal{F}_{i j k}(S+\bar{S}) \bar{F}_{\bar{S}}-D_{k}\left(\mathcal{F}_{i j \ell}\right)(S+\bar{S}) \bar{Z}_{\bar{S}}^{\ell} .
\end{aligned}
$$

Equations (4.16)-(4.23) determine much of the structure of the type IIB flux vacua. Of crucial importance for our discussion of the $\mathcal{O}(1)$ fine-tuning of the Hessian matrix in section 3.2.1 was that the lightest $X^{i}$ field was lifted at $\mathcal{O}(\epsilon)$. We noted that if $\operatorname{Re}\left(\bar{W} D_{i} Z_{j k} \bar{f}^{i} \bar{f}^{j} \bar{f}^{k}\right)$ was of $\mathcal{O}\left(\epsilon^{0}\right)$, then cross-couplings with heavier $X^{i}$ fields were negligible, and meta-stability requires the fine-tuning of $\mathcal{O}(1)$ terms. In contrast, for $\operatorname{Re}\left(\bar{W} D_{i} Z_{j k} \bar{f}^{i} \bar{f}^{j} \bar{f}^{k}\right) \sim \epsilon$ we found an additional cancellation of the remaining $\mathcal{O}(\epsilon)$ corrections, and the lightest $X^{i}$ field would be lifted at $\mathcal{O}\left(\epsilon^{2}\right)$ with a generic $\mathcal{O}(N)$ fine-tuning of the Hessian as a result. Here, we note that equation (4.23) implies that the condition of minimal $\mathcal{O}(1)$ fine-tuning of the Hessian matrix in type IIB string theory can be phrased as a geometric constraint on the field space curvature. More precisely, minimal tuning requires:

$$
D_{i}\left(\mathcal{F}_{j k \ell}\right) \bar{f}^{i} \bar{f}^{j} \bar{f}^{k} \bar{Z}_{\bar{S}}^{\ell}=\mathcal{O}\left(\epsilon^{0}\right) \quad[\text { for } \mathcal{O}(1) \text { finetuning }] .
$$

While this condition is expected to be satisfied for generic 'special geometries', it fails in some well-studied models in which the complex structure moduli space is a symmetric space with covariantly constant Riemann curvature for which $D_{k}\left(\mathcal{F}_{i j \ell}\right)=0$. As discussed in section 3.2.1, in this case the lightest $X^{i}$ field is lifted at $\mathcal{O}\left(\epsilon^{2}\right)$ together with the noscale field $T$, and cross-couplings with the heavier complex structure moduli are no longer automatically negligible. However, as we now illustrate in the case of the STU-models, even in this case one can construct examples of this type of vacua.

\subsection{The STU-model with one non-perturbative effect}

Let us exemplify our findings in the simple 'STU-model' with three complex moduli: the axio-dilaton $S=e^{-\phi}-i C_{0}$, a complex structure modulus $U$ and a complexified volume modulus $T$. This model arises by compactifying type IIB string theory on $T^{6} / \mathbb{Z}_{2} \times \mathbb{Z}_{2}$, if we restrict ourselves to the isotropic sector and take the three $T^{2}$ in the $T^{6}$ to be equal. The latter can be for example imposed by an extra $\mathbb{Z}_{3}$ symmetry that rotates the three $T^{2}$, see section 2 in [39]. In this model, the complex structure moduli space is a symmetric space, so that from our general discussion around the condition (4.24), we expect $m_{1-}^{2} \sim \epsilon^{2} m_{3 / 2}^{2}$ and that $\mathcal{O}(N)=\mathcal{O}(3)$ terms need to be fine-tuned to ensure the metastability of the lightest direction in the $S U$ subspace.

In the presence of $F_{3}$ and $H_{3}$ fluxes the resulting Kähler and superpotential are

$$
K=-3 \log (T+\bar{T})-\log (S+\bar{S})-3 \log (U+\bar{U}),
$$




$$
\begin{aligned}
W= & a_{0}+3 i a_{1} U+3 a_{2} U^{2}+i a_{3} U^{3}+S\left(i b_{0}+3 b_{1} U+3 i b_{2} U^{2}+b_{3} U^{3}\right) \\
& +W_{n p}(S, T, U),
\end{aligned}
$$

where the real coefficients $a_{i}, b_{i} \in \mathbb{Z}$ correspond to the $F_{3}$ and $H_{3}$ fluxes respectively. In particular, if we set $(2 \pi)^{2} \alpha^{\prime}=1$, then

$$
\int_{\Sigma_{i}} F_{3}=a_{i}, \quad \int_{\Sigma_{i}} H_{3}=b_{i}
$$

where the $\Sigma_{i}$ are 3-cycles in the integer homology of the orientifolded space. This leads to $\vec{N}=\vec{f}-i S \vec{h}=\vec{a}-i S \vec{b}$ and the above superpotential. The above Kähler and superpotential give rise to the usual type IIB no-scale Minkowski vacua discussed in [26]. We will below determine the parameters $a_{i}, b_{i}$ in terms of the vacuum expectation values of the moduli and the parameter $\epsilon$ (see eq. (4.31)). Plugging these into the superpotential above leads to a superpotential that contains terms up to quadratic order in $\epsilon$.

The volume modulus $T$ appears in the superpotential only through non-perturbative corrections, which arise from Euclidean D3-branes and from gaugino condensation on $D 7$-branes, and to leading order take the form $W_{n p}=A(S, U) e^{-a T}$. Naturally these nonperturbative corrections are small compared to the tree-level flux contribution so that we can think of them as a perturbation around the no-scale Minkowski vacuum and apply our general approach. Since the moduli $S$ and $U$ are stabilised by the fluxes at tree-level one can assume that the small perturbation does not shift their minimum much so we can neglect the dependence of $A$ on $S$ and $U$ and treat $A$ effectively as a constant: ${ }^{5}$

$$
W_{n p}=A e^{-a T}
$$

To simplify the equations, we set $a_{1}=a_{3}=b_{0}=b_{2}=0$. Then $W$ and all its Kähler covariant derivatives are real, if we set the imaginary parts of $S, T$ and $U$ to zero. In particular this means that we can trivially solve half of the critical point equations by setting $\operatorname{Im}(S)=\operatorname{Im}(T)=\operatorname{Im}(U)=0$.

It is convenient to solve this system by considering the 'inverse problem' of finding the fluxes which will allow for a minimum at the moduli vevs $S=S_{0}, T=T_{0}$ and $U=U_{0}$ with $S_{0}, T_{0}, U_{0} \in \mathbb{R}$. By appropriate $\operatorname{SL}(2, \mathbb{Z})$ transformations we can always ensure that $S_{0}$ and $U_{0}$ are in the fundamental domain (i.e. being larger or equal than one). This is important if we want to count $\mathrm{dS}$ vacua, but since we are only interested in their existence we refrain from explicitly doing these $\mathrm{SL}(2, \mathbb{Z})$ transformations. Furthermore, our supergravity solutions require $S_{0}>1$ and $T_{0} \gg 1$ in order to ensure that string loop and $\alpha^{\prime}$ corrections are small.

The critical point equations are solved by the $F$-terms,

$$
F_{S}=\frac{1}{4 S_{0}} W_{0}^{(\epsilon=0)} \epsilon
$$

\footnotetext{
${ }^{5}$ As we discussed above there should be one real direction among $S$ and $U$ that gets generically a mass of $\mathcal{O}(\epsilon)$ which is still sufficient to treat $A$ as constant. In the simple STU-model there are further cancellations and this light direction only gets a mass at $\mathcal{O}\left(\epsilon^{2}\right)$ so that the dependence of $A$ on this light direction could be important. It would be interesting to explicitly calculate the function $A(S, U)$ in this model to check whether it is justified to take $A$ to be constant.
} 


$$
\begin{aligned}
& F_{T}=K_{T} W+\frac{\lambda}{T_{0}} \epsilon^{2}, \\
& F_{U}=\frac{3}{4 U_{0}} W_{0}^{(\epsilon=0)} \epsilon,
\end{aligned}
$$

where $\lambda=T_{0} \delta W_{T} / \epsilon^{2}$ is a real number of order $\mathcal{O}\left(\epsilon^{0}\right)$, and where $W_{0}^{(\epsilon=0)}$ denotes the flux superpotential at the critical point to zeroth order in $\epsilon$, or equivalently, the value of $W$ at the non-supersymmetric Minkowski minimum with unbroken no-scale symmetry. The flux superpotential can be expressed in terms of $\lambda$ and $T_{0}$ as,

$$
W_{0}^{(\epsilon=0)}=\frac{4 \lambda}{3}\left(2+a T_{0}\right) .
$$

The choice of normalising the F-terms of equation (3.4) in terms of $W_{0}^{(\epsilon=0)}$ is convenient as this way the $\epsilon$-expansion of the fluxes truncates at second order:

$$
\begin{aligned}
& a_{0}=\lambda\left(-\frac{1}{6}\left(7+2 a T_{0}\right) \epsilon+\frac{\left(8+a T_{0}\left(4+a T_{0}\right)\right)}{4 a T_{0}\left(2+a T_{0}\right)} \epsilon^{2}\right), \\
& a_{2}=\frac{\lambda}{U_{0}^{2}}\left(\frac{2\left(2+a T_{0}\right)}{9}+\frac{7}{18} \epsilon+\frac{\left(8+a T_{0}\right)}{36\left(2+a T_{0}\right)} \epsilon^{2}\right), \\
& b_{1}=\frac{U_{0}}{S_{0}} a_{2}, \\
& b_{3}=\frac{\lambda}{S_{0} U_{0}^{3}}\left(\frac{\left(1+2 a T_{0}\right)}{6} \epsilon+\frac{a T_{0}}{4\left(2+a T_{0}\right)} \epsilon^{2}\right), \\
& A=-\epsilon^{2} \frac{e^{a T_{0}} \lambda}{a T_{0}} .
\end{aligned}
$$

In this particular example some fluxes turn out to be of $\mathcal{O}(\epsilon)$. This is not generic but rather due to the simplicity of this model. One might worry further that the appearance of parametrically small $\epsilon$ and $\epsilon^{2}$ terms are in tension with having quantized fluxes. This could be resolved by appropriately choosing the values $S_{0}, T_{0}, U_{0}$ and the overall scale $\lambda$. However, we do not expect such a hierarchy (or tuning) in the vacuum expectation values of the moduli to naturally arise in this simple model since it has such a limited number of flux quanta. Generic string compactifications have however very large numbers of flux parameters and should more naturally allow for a parametrically small $\epsilon$.

The $F$-terms in the $(S, U)$ subspace of equation (4.29) are aligned with the unit vector $f_{i}^{T}=\left(1 /\left(4 S_{0}\right), 3 /\left(4 U_{0}\right)\right)$ as in $(3.4)$ and section 3.2 .2 , which upon canonical normalisation is given by $f_{i}^{T}=(1 / 2, \sqrt{3} / 2)$, independently of the field vevs and fluxes. The gravitino mass at the de Sitter vacuum is to $\mathcal{O}\left(\epsilon^{0}\right)$ given by,

$$
m_{3 / 2}^{2}=e^{K}|W|^{2}=\frac{\left(2+a T_{0}\right)^{2} \lambda^{2}}{72 S_{0} T_{0}^{3} U_{0}^{3}} .
$$

We may now proceed as in section 3 to systematically extract the eigenvalues of the Hessian matrix.

The heaviest fields in the system correspond to the linear combination of $S$ and $U$ that are perpendicular to $f_{i}$. These fields are lifted by the fluxes at order $\mathcal{O}\left(\epsilon^{0}\right)$ with the 
physical masses (we define $M_{i}^{2}$ to be the eigenvalues of $V^{\prime \prime}$ in a canonically normalised basis):

$$
\begin{aligned}
& M_{2+}^{2}=\frac{16}{9} m_{3 / 2}^{2}, \\
& M_{2-}^{2}=\frac{4}{9} m_{3 / 2}^{2},
\end{aligned}
$$

up to corrections of $\mathcal{O}(\epsilon)$. In addition, one heavy real degree of freedom corresponding to the $v_{1+}$ direction of equation (3.14) is lifted at $\mathcal{O}\left(\epsilon^{0}\right)$ by the fluxes. By explicit diagonalisation, we confirm equation (3.15) to zeroth order in $\epsilon$,

$$
M_{1+}^{2}=4 m_{3 / 2}^{2} .
$$

The remaining three real degrees of freedom corresponding to $v_{1-}, \operatorname{Re}(T)$ and $\operatorname{Im}(T)$ are lifted at order $\epsilon^{2}$. From our general discussion in section 3.2.2, we expect that if the lightest $X^{i}$ field (the $v_{1}$ - direction) is lifted at $\mathcal{O}\left(\epsilon^{2}\right)$, then an $\mathcal{O}(N)$ fine-tuning is necessary in order to ensure that cross couplings with heavier $X^{i}$ fields do not destabilise the vacuum. In this STU example however, we find that such cross-terms - which we in general expect to enter at $\mathcal{O}(\epsilon)$ - are absent both at $\mathcal{O}(\epsilon)$ and $\mathcal{O}\left(\epsilon^{2}\right)$. Thus, in this particular model, the lightest $X$-field is lighter than the generic expectation, but this still does not result in an increased amount of tuning needed in order to achieve metastability.

The physical masses of the three lightest degrees of freedom are then given by,

$$
\begin{aligned}
M_{L_{1}}^{2}= & \frac{2\left(a T_{0}\right)^{2}}{\left(2+a T_{0}\right)} m_{3 / 2}^{2} \epsilon^{2}, \\
M_{L_{2} \pm}^{2}= & \frac{1}{2\left(2+a T_{0}\right)^{2}} m_{3 / 2}^{2} \epsilon^{2}\left(21-2 a^{3} T_{0}^{3}+14 a T_{0}\right. \\
& \left.\quad \pm \sqrt{4 a T_{0}\left(a T_{0}+1\right)\left(a T_{0}\left(a T_{0}\left(a T_{0}\left(a T_{0}+7\right)+27\right)+26\right)-33\right)+217}\right),
\end{aligned}
$$

The metastability condition of these de Sitter vacua (which for only $T$ being lifted at $\mathcal{O}\left(\epsilon^{2}\right)$ is given by $a T_{0}<\sqrt{2}$, cf. equation (4.5)), here translates into the marginally stricter condition $m_{L_{2 \pm}}^{2}>0$, or,

$$
1<a T_{0}<a T_{\star} \approx 1.383=\sqrt{1.914}
$$

where $a T_{\star}$ is given by equation (A.20) in the appendix.

The cosmological constant for these de Sitter vacua is given by,

$$
V=\frac{m_{3 / 2}^{2} \epsilon^{2}}{\left(2+a T_{0}\right)^{2}}\left(\left(a T_{0}-1\right)\left(2+a T_{0}\right)-3 \epsilon-\frac{3}{4} \epsilon^{2}\right) .
$$

We want to point out that our general analysis provides sufficient and necessary conditions only for $\epsilon \ll 1$ and if all quantities scale with $\epsilon$ in the way we assumed. For larger $\epsilon$ or at points in moduli space where the scaling is different, it is very well possible that $\mathrm{dS}$ vacua still exist and their features can deviate from our predictions. Since for simple models we can find explicit, analytic families of $\mathrm{dS}$ vacua it might be possible to map out 
the entire parameter space of stable dS vacua that are connected to our analytic families. While we leave this interesting task for the future, we give one simple example for the STU-model that shows that

- stable dS vacua also exist when we move away from the regime of validity of our general ansatz.

- we can find examples with correctly quantised fluxes.

- we can choose the flux numbers so small that even in simple models it is possible to satisfy the tadpole condition.

In particular, if we take the superpotential to be

$$
W=-2+18 U^{2}+S\left(6 U+2 U^{3}\right)-3 e^{-a T},
$$

then we find stable dS vacua with $S_{0} \approx 2.02, U_{0} \approx .67$ and $a T_{0} \approx 1.73$. In particular $a T_{0}>\sqrt{2}$ which disagrees with our general prediction. We can also explicitly calculate the F-terms and find that $\left\|\left(F_{S}, 0, F_{U}\right)\right\| / W \approx 0.72$ is not small and we cannot view this solution directly as a small $\epsilon$-deformation of a no-scale Minkowski vacuum. Similarly, $\left|\delta W_{T} / W\right|$ is not small.

The integer fluxes in the superpotential (4.40) are $a_{0}=-2, a_{2}=6, b_{1}=2, b_{3}=2$, which leads to the tadpole condition (cf. for example [40])

$$
\frac{1}{2}\left(a_{0} b_{3}+3 a_{2} b_{1}\right)=\frac{1}{2}(-4+36)=\frac{1}{4} N_{O 3}=16,
$$

where the factor $1 / 2$ in front of the fluxes is due to the fact that the above tadpole condition is derived in the covering space, before taking into account the orientifold projection. We see that we exactly satisfy the tadpole condition in our simple example.

There are additional tadpole constraints due to the presence of O7-planes. The O7plane charges and tensions can be canceled by D7-branes, which also need to be present to generate the non-perturbative correction to the flux superpotential. It would be very interesting to study this in detail.

\section{Conclusions}

We have shown that $\mathcal{N}=1$ supergravities in which one of the fields has a Kähler potential of no-scale type admit de Sitter solutions that can be rendered metastable upon the tuning of only two parameters, independently of the number of chiral fields, $N$. These vacua arise from small superpotential perturbations to the well-known non-supersymmetric no-scale Minkowski vacuum. The perturbation induces small F-terms in the directions perpendicular to the no-scale modulus, $F_{i}=\mathcal{O}(\epsilon W)$. No-scale vacua that can be perturbatively lifted to de Sitter space have one real massless mode along the $F_{i}$ direction in field space, in addition to the massless no-scale modulus. This massless mode appears as a direct consequence of the critical point equation and extends the theorem of [28] for non-supersymmetric deformations of supersymmetric Minkowski solutions to have at least one real flat direction. 
At the de Sitter critical point, the lingering approximate no-scale structure of the Hessian matrix ensures that $2 N-3$ real modes receive positive definite eigenvalues that are (at least) of the order of the gravitino mass, $m_{3 / 2}=e^{K / 2}|W|$. The remaining three modes, which we denote ' $v_{1-}$ ' and ' $T \pm$ ', are lifted at $\mathcal{O}(\epsilon)$ and $\mathcal{O}\left(\epsilon^{2}\right)$, respectively. In general, the stabilisation of such light modes require fine-tuning of off-diagonal Hessian cross-terms with heavier modes, however, in section 3.2.2 we found that the no-scale structure again leads to a cancellation of the leading order cross-terms, thus reducing the question of the full stabilisation of the vacuum to that of two $2 \times 2$ matrices. Metastability can then be obtained by tuning the ratio of the off-diagonal entries to the diagonal entries of these matrices, which we showed corresponds to requiring

$$
\operatorname{Re}\left(\bar{W} D_{i} Z_{j k} \bar{f}^{i} \bar{f}^{j} \bar{f}^{k}\right)>0
$$

and ensuring that

$$
-\frac{4}{3} K_{T} \operatorname{Re}\left(\bar{W} \delta W_{T}\right)-\left|K^{T} \bar{W} \delta W_{T T T}-\frac{4}{3} K_{T} \operatorname{Re}\left(\bar{W} \delta W_{T}\right)\right|>0 .
$$

With this moderate tuning, the spectrum of the lightest modes is given by,

$$
m_{1-}^{2} \sim m_{3 / 2}^{2} \epsilon>0, \quad m_{T \pm}^{2} \sim m_{3 / 2}^{2} \epsilon^{2}>0 .
$$

We stress that this mechanism for the decoupling of many modes is different from the well-known supersymmetric decoupling, in which many fields are given positive definite supersymmetric squared masses at some scale $m_{\text {susy }}^{2} \gg m_{3 / 2}^{2}$. In contrast, the 'decoupling by no-scale' developed in this paper does not require large (or small) supersymmetric masses for any of the fields, but is rather ensured by the very particular no-scale structure. For approximate no-scale de Sitter vacua, the (classical) vacuum energy is hierarchically smaller than the generic supergravity expectation,

$$
\langle V\rangle \sim m_{3 / 2}^{2} M_{\mathrm{Pl}}^{2} \epsilon^{2} \ll m_{3 / 2}^{2} M_{\mathrm{Pl}}^{2} .
$$

Since the no-scale form of the Kähler potential often appears in the four dimensional effective theories derived from dimensional reduction of string theory, we expect that the general scenario presented in this paper is relevant for constructing metastable de Sitter vacua in string theory. In section 4.3 , we considered the embedding of this scenario in the type IIB 'landscape' of flux compactifications, in which fine-tuning of three-form fluxes can ensure that $\epsilon \ll 1$. We derived a geometric condition on the complex structure field space geometry for the realisation of the minimal version of this scenario with $\mathcal{O}(1)$ finetuning. We expect this condition to be satisfied for generic Calabi-Yau compactifications, but we note that it fails in toroidal orientifold compactifications in which the complex structure moduli space is a symmetric space. However, also in this case metastable de Sitter minima can be found, and in section 4.4 we explicitly constructed such solutions in STU-supergravity models.

The simplest examples of the mechanism for obtaining metastable de Sitter vacua presented in this paper have two obvious short-comings. First, if the single no-scale modulus considered in this paper is stabilised by a single non-perturbative effect with $\delta W=$ 
$A \exp (-a T)$, then metastable de Sitter solutions are only obtainable for $1<a \operatorname{Re}(T)<\sqrt{2}$ and $0.639 \epsilon^{2}<\left|A / W_{0}\right|<0.680 \epsilon^{2}$. While gaugino condensation on large rank gauge groups gives $a \ll 1$, so that $\mathcal{V} \sim(\operatorname{Re}(T))^{3 / 2} \gg 1$ and $m_{3 / 2} \ll M_{\mathrm{Pl}}$ are consistently achievable, it remains to be established that the compactification volumes can be made large enough to neglect all $\alpha^{\prime}$ corrections. Moreover, $A$ is in general a modulus dependent function, and we have not fully assessed the severity of the constraint for $\left|A / W_{0}\right|$ in string theory models. Second, the mass of the lightest two moduli is comparable to the (classical) vacuum Hubble parameter, and this may complicate the construction of viable cosmologies for these vacua.

However, while in this paper we have considered single no-scale modulus theories and in particular focused on the special case in which the no-scale symmetry is broken by a single non-perturbative superpotential correction, we know of no reason why the general mechanism presented in this paper should not be extendable to more general theories in which these shortcomings may be overcome. For example, it will be interesting to embed this mechanism in the Large Volume Scenario, which has a multiple moduli noscale sector and in which the no-scale symmetry is broken by perturbative $\alpha^{\prime}$ corrections and non-perturbative superpotential corrections, so that exponentially large volumes are obtainable $[6,7]$. There are good reasons to believe that such embeddings can successfully be achieved: small Kähler deformations of no-scale models give a positive diagonal term in the Hessian along the sGoldstino direction [41], and the approximately no-scale 'Kähler uplifting' scenarios such as those outlined in $[10,42,43]$ exhibit a similar mass scaling for the no-scale field (quadratic in the perturbation) as in our solutions (5.3).

In sum, we anticipate that the methods and results presented in this paper can be usefully applied to find fully controlled, simple de Sitter vacua in string theory. The explicit construction of such vacua is an important problem for the future.

\section{Acknowledgments}

We would like to thank J. Conlon, A. Guarino, A. Hebecker, S. Kachru, R. Kallosh, S. Krippendorf, A. Linde, A. Lukas, L. McAllister, X. de la Ossa, D. Roest, M. Rummel, G. Shiu and A. Westphal for illuminating discussions. We are particularly grateful to R. Kallosh and S. Krippendorf for comments on a draft of this paper. D.M. is funded by the European Research Council starting grant "Supersymmetry Breaking in String Theory", but the contents of this paper reflect only the authors views and not necessarily the views of the European Commission. B.V. is supported by the European Commission through the Marie Curie Intra-European fellowship 328652-QM-sing. T.W. is supported by a Research Fellowship (Grant number WR 166/1-1) of the German Research Foundation (DFG). DM is very grateful to Birzeit University for kind hospitality while finishing this paper.

\section{A Useful formulae}

We often use subscripts to indicate scalar derivatives, for example $\partial_{a} V \equiv V_{a}$. The potential and its derivatives at a critical point with $V_{a}=0$ are [34]:

$$
V=e^{K}\left(F_{a} \bar{F}^{a}-3|W|^{2}\right)
$$




$$
\begin{aligned}
V_{a} & =e^{K}\left(\left(D_{a} D_{b} W\right) \bar{F}^{b}-2 F_{a} \bar{W}\right)=e^{K}\left(Z_{a b} \bar{F}^{b}-2 F_{a} \bar{W}\right) \\
V_{a b} & =e^{K}\left(\left(D_{a} D_{b} D_{c} W\right) \bar{F}^{c}-\left(D_{a} D_{b} W\right) \bar{W}\right)=e^{K}\left(U_{a b c} \bar{F}^{c}-Z_{a b} \bar{W}\right) \\
V_{a \bar{b}} & =e^{K}\left(-R_{a \bar{b} c \bar{d}} \bar{F}^{c} F^{\bar{d}}+K_{a \bar{b}} F_{c} \bar{F}^{c}-F_{a} \bar{F}_{\bar{b}}+\left(D_{a} D_{c} W\right)\left(\bar{D}_{\bar{b}} \bar{D}^{c} \bar{W}\right)-2 K_{a \bar{b}}|W|^{2}\right. \\
& =e^{K}\left(-R_{a \bar{b} c \bar{d}} \bar{F}^{c} F^{\bar{d}}+K_{a \bar{b}} F_{c} \bar{F}^{c}-F_{a} \bar{F}_{\bar{b}}+(Z \bar{Z})_{a \bar{b}}-2 K_{a \bar{b}}|W|^{2}\right.
\end{aligned}
$$

where we used the definitions $F_{a}=D_{a} W, Z_{a b}=D_{a} F_{b}$ and $U_{a b c}=D_{a} Z_{b c}$ and $D_{a}$ is the Kähler and diffeomorphism covariant derivative such that for example $Z_{a b}=\partial_{a} F_{b}+K_{a} F_{b}-$ $\Gamma_{a b}^{c} F_{c}$.

The derivatives of our Kähler potential with respect to the no-scale modulus are:

$$
\begin{aligned}
K & =-3 \ln (T+\bar{T})+\tilde{K}\left(X^{i} \bar{X}^{i}\right), \\
\frac{1}{3} K_{T} & =\frac{1}{K^{T}} \\
K_{T \bar{T}} & =\frac{1}{3} K_{T} \bar{K}_{\bar{T}}, \\
K_{T} K_{T \bar{T}} & =\frac{3}{2} K_{T \bar{T} T}, \\
\Gamma_{T T}^{T} & =\frac{2}{3} K_{T}, \\
R_{T \bar{T} T \bar{T}} & =\frac{2}{3} K_{T \bar{T}} K_{T \bar{T}} .
\end{aligned}
$$

In our conventions the Kähler potential is symmetric under $T$ and $\bar{T}$ exchange so that derivatives with respect to $T$ and $\bar{T}$ are the same, for example $K_{T T}=K_{T \bar{T}}=K_{\overline{T T}}$.

Straightforward calculations lead to the following covariant derivatives:

$$
\begin{aligned}
W & =W_{0}\left(X^{i}\right)+\delta W\left(X^{i}, T\right), \\
F_{T} & =K_{T} W+\delta W_{T}, \\
F_{i} & =K_{i} W+W_{i}, \\
Z_{T T} & =\delta W_{T T}+\frac{4}{3} K_{T} \delta W_{T}+2 K_{T T} W \\
Z_{T i} & =\delta W_{T i}+K_{i} \delta W_{T}+K_{T} F_{i}, \\
Z_{i j} & =W_{i j}+K_{i} F_{j}+K_{j} F_{i}-\Gamma_{i j}^{k} F_{k}+\left(K_{i j}-K_{i} K_{j}\right) W \\
U_{T T T} & =K_{T T T} W+\delta W_{T T T}+K_{T} \delta W_{T T}+2 K_{T T} \delta W_{T}, \\
U_{T T i} & =2 K_{T T} F_{i}+\partial_{T T}^{2} F_{i}+\frac{4}{3} K_{T} \partial_{T} F_{i} \\
& =2 K_{T T}\left(K_{i} W+W_{i}\right)+K_{i} \delta W_{T T}+\delta W_{T T i}+\frac{4}{3} K_{T}\left(K_{i} \delta W_{T}+\delta W_{T i}\right), \\
U_{i j T} & =\left(\partial_{T}+K_{T}\right) Z_{i j}=K_{T} Z_{i j}+\delta W_{T i j}+K_{i} \delta W_{T j}+K_{j} \delta W_{T i}-\Gamma_{i j}^{k} \delta W_{T k} \\
\quad & +\left(K_{i j}+K_{i} K_{j}-\Gamma_{i j}^{k} K_{k}\right) \delta W_{T} .
\end{aligned}
$$




\section{A.1 STU-model}

The requirement $M_{L_{2}-}^{2}>0$ for the squared masses of (4.38) determines the upper bound $a T_{0}<a T_{\star}$ with,

$$
\begin{aligned}
a T_{\star} & =\frac{1}{12}\left(-12+\sqrt{3(38+c)}+\left[228-3 c+576 \sqrt{\frac{3}{38+c}}\right]^{\frac{1}{2}}\right) \approx 1.383, \\
c & =\sqrt[3]{53477-108 \sqrt{105389}}+\sqrt[3]{53477+108 \sqrt{105389}} .
\end{aligned}
$$

Open Access. This article is distributed under the terms of the Creative Commons Attribution License (CC-BY 4.0), which permits any use, distribution and reproduction in any medium, provided the original author(s) and source are credited.

\section{References}

[1] Supernova Search Team collaboration, A.G. Riess et al., Observational evidence from supernovae for an accelerating universe and a cosmological constant, Astron. J. 116 (1998) 1009 [astro-ph/9805201] [INSPIRE].

[2] Supernova Cosmology Project collaboration, S. Perlmutter et al., Measurements of $\Omega$ and $\Lambda$ from 42 high redshift supernovae, Astrophys. J. 517 (1999) 565 [astro-ph/9812133] [INSPIRE].

[3] N. Suzuki et al., The Hubble Space Telescope cluster supernova survey: V. Improving the dark energy constraints above $z>1$ and building an early-type-hosted supernova sample, Astrophys. J. 746 (2012) 85 [arXiv:1105.3470] [INSPIRE].

[4] Planck collaboration, P.A.R. Ade et al., Planck 2013 results. XVI. Cosmological parameters, Astron. Astrophys. 571 (2014) A16 [arXiv:1303.5076] [INSPIRE].

[5] S. Kachru, R. Kallosh, A.D. Linde and S.P. Trivedi, De Sitter vacua in string theory, Phys. Rev. D 68 (2003) 046005 [hep-th/0301240] [INSPIRE].

[6] V. Balasubramanian, P. Berglund, J.P. Conlon and F. Quevedo, Systematics of moduli stabilisation in Calabi-Yau flux compactifications, JHEP 03 (2005) 007 [hep-th/0502058] [INSPIRE].

[7] J.P. Conlon, F. Quevedo and K. Suruliz, Large-volume flux compactifications: moduli spectrum and D3/D7 soft supersymmetry breaking, JHEP 08 (2005) 007 [hep-th/0505076] [INSPIRE].

[8] K. Sousa and P. Ortiz, Perturbative stability along the supersymmetric directions of the landscape, JCAP 02 (2015) 017 [arXiv: 1408.6521] [INSPIRE].

[9] U.H. Danielsson et al., De Sitter hunting in a classical landscape, Fortsch. Phys. 59 (2011) 897 [arXiv: 1103.4858] [INSPIRE].

[10] M. Rummel and A. Westphal, A sufficient condition for de Sitter vacua in type IIB string theory, JHEP 01 (2012) 020 [arXiv:1107.2115] [INSPIRE].

[11] M. Cicoli, A. Maharana, F. Quevedo and C.P. Burgess, De Sitter string vacua from dilaton-dependent non-perturbative effects, JHEP 06 (2012) 011 [arXiv:1203.1750] [INSPIRE]. 
[12] M. Cicoli, S. Krippendorf, C. Mayrhofer, F. Quevedo and R. Valandro, D-branes at del Pezzo singularities: global embedding and moduli stabilisation, JHEP 09 (2012) 019 [arXiv: 1206.5237] [INSPIRE].

[13] A. Hebecker, S.C. Kraus, M. Kuntzler, D. Lüst and T. Weigand, Fluxbranes: moduli stabilisation and inflation, JHEP 01 (2013) 095 [arXiv:1207.2766] [INSPIRE].

[14] J. Louis, M. Rummel, R. Valandro and A. Westphal, Building an explicit de Sitter, JHEP 10 (2012) 163 [arXiv:1208.3208] [INSPIRE].

[15] U. Danielsson and G. Dibitetto, On the distribution of stable de Sitter vacua, JHEP 03 (2013) 018 [arXiv: 1212.4984] [INSPIRE].

[16] J. Blåbäck, U. Danielsson and G. Dibitetto, Fully stable dS vacua from generalised fluxes, JHEP 08 (2013) 054 [arXiv:1301.7073] [INSPIRE].

[17] C. Damian, L.R. Diaz-Barron, O. Loaiza-Brito and M. Sabido, Slow-roll inflation in non-geometric flux compactification, JHEP 06 (2013) 109 [arXiv:1302.0529] [INSPIRE].

[18] C. Damian and O. Loaiza-Brito, More stable de Sitter vacua from S-dual nongeometric fluxes, Phys. Rev. D 88 (2013) 046008 [arXiv:1304.0792] [InSPIRE].

[19] M. Cicoli et al., Explicit de Sitter flux vacua for global string models with chiral matter, JHEP 05 (2014) 001 [arXiv:1312.0014] [INSPIRE].

[20] J. Blåbäck, D. Roest and I. Zavala, De Sitter vacua from nonperturbative flux compactifications, Phys. Rev. D 90 (2014) 024065 [arXiv: 1312.5328] [INSPIRE].

[21] U. Danielsson and G. Dibitetto, An alternative to anti-branes and O-planes?, JHEP 05 (2014) 013 [arXiv: 1312.5331] [INSPIRE].

[22] M. Rummel and Y. Sumitomo, De Sitter vacua from a D-term generated racetrack uplift, JHEP 01 (2015) 015 [arXiv: 1407.7580] [INSPIRE].

[23] S. Ferrara, R. Kallosh and A. Linde, Cosmology with nilpotent superfields, JHEP 10 (2014) 143 [arXiv: 1408.4096] [INSPIRE].

[24] R. Kallosh and A. Linde, Inflation and uplifting with nilpotent superfields, JCAP 01 (2015) 025 [arXiv: 1408.5950] [INSPIRE].

[25] R. Kallosh and T. Wrase, Emergence of spontaneously broken supersymmetry on an anti-D3-brane in KKLT dS vacua, JHEP 12 (2014) 117 [arXiv:1411.1121] [INSPIRE].

[26] S.B. Giddings, S. Kachru and J. Polchinski, Hierarchies from fluxes in string compactifications, Phys. Rev. D 66 (2002) 106006 [hep-th/0105097] [INSPIRE].

[27] A. Saltman and E. Silverstein, The scaling of the no scale potential and de Sitter model building, JHEP 11 (2004) 066 [hep-th/0402135] [INSPIRE].

[28] R. Kallosh, A. Linde, B. Vercnocke and T. Wrase, Analytic classes of metastable de Sitter vacua, JHEP 10 (2014) 011 [arXiv:1406.4866] [INSPIRE].

[29] D. Marsh, L. McAllister and T. Wrase, The wasteland of random supergravities, JHEP 03 (2012) 102 [arXiv:1112.3034] [INSPIRE].

[30] E. Cremmer, S. Ferrara, C. Kounnas and D.V. Nanopoulos, Naturally vanishing cosmological constant in $N=1$ supergravity, Phys. Lett. B 133 (1983) 61 [INSPIRE].

[31] D.V. Nanopoulos, The march towards no scale supergravity, NATO Adv. Study Inst. Ser. B 352 (1996) 677 [hep-ph/9411281] [INSPIRE]. 
[32] F. Denef and M.R. Douglas, Distributions of flux vacua, JHEP 05 (2004) 072 [hep-th/0404116] [INSPIRE].

[33] R. Brustein and S.P. de Alwis, Moduli potentials in string compactifications with fluxes: mapping the discretuum, Phys. Rev. D 69 (2004) 126006 [hep-th/0402088] [INSPIRE].

[34] F. Denef and M.R. Douglas, Distributions of nonsupersymmetric flux vacua, JHEP 03 (2005) 061 [hep-th/0411183] [INSPIRE].

[35] M. Haack, D. Krefl, D. Lüst, A. Van Proeyen and M. Zagermann, Gaugino condensates and D-terms from D7-branes, JHEP 01 (2007) 078 [hep-th/0609211] [INSPIRE].

[36] I. Antoniadis, K.S. Narain and T.R. Taylor, Higher genus string corrections to gauge couplings, Phys. Lett. B 267 (1991) 37 [INSPIRE].

[37] T. Bachlechner, M. Marsh, L. McAllister and T. Wrase, unpublished notes.

[38] J. Bausch, On the efficient calculation of a linear combination of chi-square random variables with an application in counting string vacua, J. Phys. A 46 (2013) 505202 [arXiv: 1208.2691] [INSPIRE].

[39] J. Shelton, W. Taylor and B. Wecht, Nongeometric flux compactifications, JHEP 10 (2005) 085 [hep-th/0508133] [INSPIRE].

[40] A. Font, A. Guarino and J.M. Moreno, Algebras and non-geometric flux vacua, JHEP 12 (2008) 050 [arXiv:0809.3748] [INSPIRE].

[41] L. Covi et al., De Sitter vacua in no-scale supergravities and Calabi-Yau string models, JHEP 06 (2008) 057 [arXiv:0804.1073] [INSPIRE].

[42] V. Balasubramanian and P. Berglund, Stringy corrections to Kähler potentials, SUSY breaking and the cosmological constant problem, JHEP 11 (2004) 085 [hep-th/0408054] [INSPIRE].

[43] A. Westphal, De Sitter string vacua from Kähler uplifting, JHEP 03 (2007) 102 [hep-th/0611332] [INSPIRE]. 